\title{
EFFECT OF COMPLEXATION CONDITIONS ON MICROCAPSULATION OF LACTOBACILLUS ACIDOPHILUS IN XANTHAN-CHITOSAN POLYELECTROLYTE COMPLEX GELS
}

\author{
He Chen ${ }^{1}$, Yajuan Song ${ }^{1}$, Nina Liu, Hongchang Wan², Guowei Shu ${ }^{1 凶}$, Na Liao \\ ${ }^{1}$ College of Life Science and Engineering, Shaanxi University of Science and Technology \\ Xi'an, 710021, China \\ ${ }^{2}$ Shaanxi Yatai Dairy Co., Ltd. \\ Xianyang, 713701, China
}

\begin{abstract}
Background. Lactobacillus acidophilus has become increasingly popular because of their beneficial effects on health of their host, and are called proboscis. In order to exert beneficial effects for probiotics, they must be able to tolerate the acidic conditions of the stomach environment and the bile in the small intestine. Microencapsulated form has received reasonable attention, since it can protect probiotic organisms against an unfavourable environment, and to allow their release in a viable and metabolically active state in the intestine. The aim of this study was to investigate some factores, such as chitosan solution $\mathrm{pH}$ and concentration, xanthan concentration, cell suspension-xanthan ratio, mixed bacteria glue liquid-chitosan ratio, which impacted the process of microencapsulation of $L$. acidophilus.

Material and methods. In this study, L. acidophilus was immobilized with xanthan/chitosan gel using extrusion method. The viable counts and encapsulation yield of $L$. acidophilus encapsulated in different chitosan solution $\mathrm{pH}(4.5,5,5.5$ and 6$)$, in different chitosan concentration $(0.5 \%, 0.7 \%, 0.9 \%$ and $1.1 \%)$, in different xanthan concentration $(0.5 \%, 0.7 \%, 0.9 \%$ and $1.1 \%)$, in different cell suspension-xanthan ratios $(1: 5,1: 10$, $1: 15$ and 1:20), in different mixed bacteria glue liquid-chitosan ratios (1:3, 1:4, 1:5 and 1:6), have been investigated by single factor experiment method.

Results. The optimum conditions of microencapsulated $L$. acidophilus have been observed. The optimum chitosan solution $\mathrm{pH}$ for L. acidophilus was 5.5; the optimum chitosan concentration was $0.9 \%$; the optimum xanthan concentration was $0.7 \%$; the optimum cell suspension-xanthan ratio was 1:10; the optimum mixed bacteria glue liquid-chitosan ratio was 1:3.

Conclusions. These results will be helpful to further optimize the process of L. acidophilus microencapsulation, and provide reference for obtaining higher viable counts and entrapped yield of L. acidophilus microcapsules.
\end{abstract}

Key words: xanthangum, chitosan, Lactobacillus acidophilus, microencapsulation, extrusion

\footnotetext{
*The project was supported by Science and Technology Research Development plan project of Shaanxi Province, China (no. 2014K01-17-07), Undergraduate Training Programs for Innovation and Entrepreneurship of Shaanxi Province (no. 1057).

\shuguowei@gmail.com 


\section{INTRODUCTION}

Considering the increasing demand for functional foods, probiotics have become one of the most important health promoting food enhancement in recent years (Agrawal, 2005). Probiotics are viable microorganisms which are beneficial to the host when administered in adequate amounts (Xiao et al., 2011).

Lactobacillus acidophilus have become increasingly popular because of their beneficial effects on the health of their host, and are called proboscis (Fuller, 1989; Motohiro Shima et al., 2009). In order to exert beneficial effects for probiotics, they must be able to tolerate the acidic conditions of the stomach environment and the bile in the small intestine (Doleyres et al., 2004; Gardiner et al., 2000). The acidic environment of the stomach and the bile salts secreted into the duodenum are the main obstacles for the survival of the ingested bacteria. Unluckily, most of the probiotics including $L$. acidophilus lack the ability to survive in the harsh conditions of acidity and bile concentration commonly encountered in the gastrointestinal tract of humans (Krasaekoopt et al., 2003; Xiao Yan Li et al., 2011). As a result, the viable counts of $L$. acidophilus probiotic dairy products often have a exponential curve downward trend, which leads to the fact that those products have a difficultly to achieve the healthy effect (Kimoto et al., 2000; Vinderola and Reinheimer, 2003).

Microencapsulated form has received reasonable attention, since it can protect probiotic organisms against an unfavourable environment, and to allow their release in a viable and metabolically active state in the intestine (Heidebach et al., 2009; Sandoval-Castilla et al., 2010). In the process of microencapsulation of probiotics, coatings and mixtures of suitable biopolymers, such as alginate, k-carrageenan, gellan-gum are applied (Champagne et al., 1994; Picot and Lacroix, 2004). However, those embedding materials have some defects in the process of microencapsulated probiotics, e.g. alginate is not stable in acidic conditions.

Chitosan is a natural biological macromolecule, which has excellent biological properties such as biocompatibility, biodegradability, lack of toxicity, and so on (Dutta et al., 2004; Felse and Panda, 1999). Xanthan gum is stable over a wide range of temperatures and $\mathrm{pH}$, which finds many applications in food (Garcia-Ochoa et al., 2000; Meyer et al., 1993).
The hydrogel network formed through the ionic interactions between the amino groups of chitosan and carboxyl groups of xanthan shows $\mathrm{pH}$-sensitive swelling characteristics, which enable the controlled release of entrapped materials such as therapeutic agents, enzymes and bacteria (Chellat et al., 2000; Chu et al., 1996). Therefore, xanthan-chitosan hydrogels are recognised as promising candidates for targeted delivery and controlled release of encapsulated products for oral administration.

In this study, L. acidophilus was immobilized with xanthan/chitosan gel using extrusion method. Some factors, such as chitosan solution $\mathrm{pH}$ and concentration, xanthan concentration, cell suspension-xanthan ratio, mixed bacteria glue liquid-chitosan ratio have been investigated. The optimum conditions of microencapsulated $L$. acidophilus will be further observed. The obtained results will be helpful to optimize the process of $L$. acidophilus microencapsulation, and provide reference for obtaining higher viable counts and entrapped yield of $L$. acidophilus microcapsules.

\section{MATERIAL AND METHODS}

\section{Preparation of chitosan and xanthan solutions}

Chitosan with a minimum of $80 \sim 95 \%$ deacetylation and a molecular weight of 370000 was purchased from Sigma-Aldrich Chemicals (St. Louis, MO). The chitosan was dissolved in $1 \mathrm{~N} \mathrm{HCl}$ by agitating, and adjusting the different chitosan concentration to $0.5 \%$, $0.7 \%, 0.9 \%$ and $1.1 \%$. The desired solution $\mathrm{pH}$ was adjusted by $1 \mathrm{M} \mathrm{NaOH}$ and deionized (DI) water was added to bring it to the final volume, and the $\mathrm{pH}$ was adjusted to $4.5,5,5.5$ and 6 . Xanthan gum with a molecular weight of 1.02 million was kindly supplied by Zibo Zhongxuan biological chemistry company. Xanthan gum was dissolved in DI water under heating and agitation, and the xanthan concentration was adjusted to $0.5 \%, 0.7 \%, 0.9 \%$ and $1.1 \%$. It was autoclaved $\left(110^{\circ} \mathrm{C}, 15 \mathrm{~min}\right)$ before use.

\section{Microorganism}

L. acidophilus was obtained from College of Life Science and Engineering, Shaanxi University of Science and Technology. One milliliter of hydrated L. acidophilus cells was inoculated into $18 \mathrm{~mL}$ MRS broth and incubated at $35^{\circ} \mathrm{C}$ for $24 \mathrm{~h}$. Actively growing cells 
were recovered from the MRS broth by centrifuging at $10000 \mathrm{rpm}$ for $15 \mathrm{~min}$. DI water was added to the cell pellet and vortexed.

\section{Microencapsulation}

L. acidophilus cells were mixed with xanthan gum solution (1:10) and encapsulation was achieved by dropwise addition of this mixture $(11 \mathrm{~mL})$ into the chitosan solution $(66 \mathrm{~mL})$ by using a manually operated syringe with $0.7-\mathrm{mm}$ cannula (BectoneDick-inson, Franklin Lakes, NJ). The chitosan solution was agitated continuously for 40 min to allow crosslinking and to avoid coales-cence of the capsules. The capsules were filtered through a $160-\mathrm{mm}$, washed twice with DI water.

\section{Viable counts}

The sample to be tested with sterile saline solution into the bacterial suspension, was next diluted at 10 times, taking the dilution of $10^{-7}$ to $10^{-8}$ of the suspension inoculation of $0.1 \mathrm{~mL}$ to the solid culture medium. After the bacteria were cultured for $48 \mathrm{~h}$ at $37^{\circ} \mathrm{C}$, we could observe and count the average values, and investigate the various factors on the microencapsulation of L. acidophilus viable counts. The viable counts of microcapsules were weighed through a formula according to eq. (1):

$$
V C=N \cdot T \cdot 10
$$

where:

$V C$ - viable counts of the original suspension a per milliliter, $\mathrm{CFU} \cdot \mathrm{mL}^{-1}$,

$N$ - average colony number of 3 repeat solid culture in the same dilution, $\mathrm{CFU} \cdot \mathrm{mL}^{-1}$,

$T$ - times of dilution.

\section{Encapsulation yield (EY)}

$1 \mathrm{~g}$ capsules were subjected to the simulated gastric fluid $(10 \mathrm{~mL})$ at $\mathrm{pH} 2.0$ for $40 \mathrm{~min}$ at $37^{\circ} \mathrm{C}$ under 210 rpm.

$$
\begin{gathered}
E Y=N_{1} \cdot M /\left(N_{0} \cdot V_{0}\right) \cdot 100 \% \\
N_{1}=G_{1} \cdot V_{1}
\end{gathered}
$$

where:

$G_{1}$ - the number of viable counts released from the simulated gastric fluid, $1 \mathrm{~mL}$,
$V_{1}$ - the volume of simulated gastric fluid, $\mathrm{mL}$,

$N$ - the living bacteria number, in wet capsule, $\mathrm{CFU} \cdot \mathrm{g}^{-1}$,

$N_{0}$ - the viable counts of the original suspension on a per milliliter, $\mathrm{CFU} \cdot \mathrm{mL}^{-1}$,

$V_{0}$ - the volume of original bacteria liquid which used by preparing microcapsule, $\mathrm{mL}$,

$M$ - the total weight of the wet capsule, g.

\section{RESULTS AND DISCUSSION}

\section{Effect of chitosan solution pH on encapsulation of $L$. acidophilus}

According to the initial preparation conditions of microcapsulation, chitosan solution $\mathrm{pH}$ was adjusted to 4.5, 5.0, 5.5 and 6, the results as shown in Figure 1.

According to Figure 1, with increasing of the chitosan solution $\mathrm{pH}$, the viable counts and entrapped yield of L. acidophilus microcapsules increased at first, and then decreased. When the chitosan solution $\mathrm{pH}$ was 5.5, the viable counts and entrapped yield of L. acidophilus microcapsules up to $1.21 \cdot 10^{10} \mathrm{CFU} \cdot \mathrm{g}^{-1}$ and $78 \%$, respectively. The reason of this tendency on figure was that the change of the chitosan solution $\mathrm{pH}$, since chitosan solution $\mathrm{pH}$ continually increased, the amino groups became less charged, which lead to fewer ionic linkages would occur between the two polymers. As a result, the crosslinking densities of xanthan-chitosan hydrogel capsules decreased, diffusion

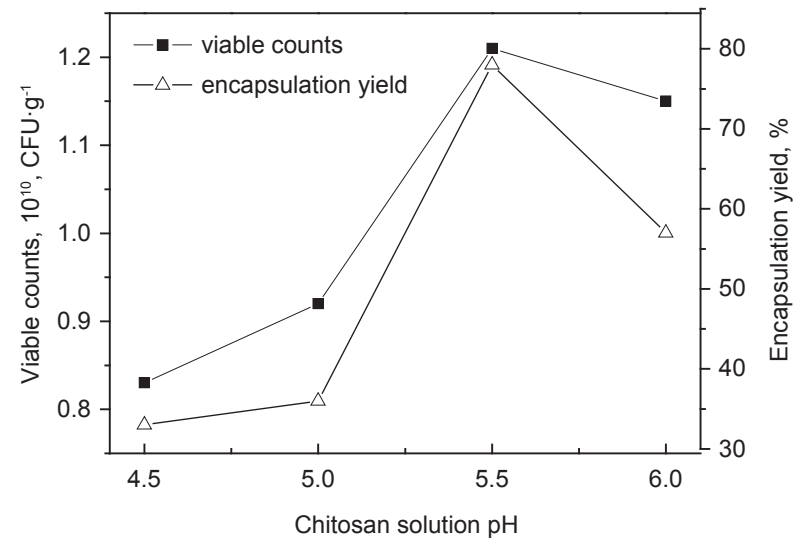

Fig. 1. Effect of chitosan solution $\mathrm{pH}$ on viable counts and entrapped yield of $L$. acidophilus of microcapsules 
coefficient became higher for chitosan chains, so amounts of bacteria spread out from xanthan-chitosan hydrogel capsules, viable counts of encapsulation and encapsulation yield of $L$. acidophilus microcapsules will be reduced, correspondingly.

As a result, there is a preliminary determination about the chitosan solution $\mathrm{pH}$ for $L$. acidophilus microencapsulated. The optimum chitosan solution $\mathrm{pH}$ was 5.5 , which corresponds to viable counts and entrapped yield were $1.21 \cdot 10^{10} \mathrm{CFU} \cdot \mathrm{g}^{-1}$ and $78 \%$, respectively.

\section{Effect of chitosan concentration on encapsulation of $L$. acidophilus}

According to the initial preparation conditions of microcapsulation, chitosan concentration was adjusted to $0.5 \%, 0.7 \%, 0.9 \%$ and $1.1 \%$, the results as shown in Figure 2.

According to Figure 2, with increasing of the chitosan concentration, the viable counts and entrapped yield of L. acidophilus microcapsules increased at first, and then decreased. When the chitosan concentration was $0.9 \%$, the viable counts and entrapped yield of L. acidophilus microcapsules up to $1.1 \cdot 10^{10} \mathrm{CFU} \cdot \mathrm{g}^{-1}$ and $73 \%$, respectively. Since chitosan concentration increased, the amino groups became more charged, which lead to more ionic linkages would occur between the two polymers. As a result, the crosslinking densities of xanthan-chitosan hydrogel capsules increased, amounts of bacteria would be embedded completely, viable counts of encapsulation and encapsulation yield of $L$. acidophilus microcapsules would be increased, correspondingly. However, when the chitosan concentration continually increased, the viscosity of chitosan solution correspondingly increased, which impeded the positive and negative charges to combine, resulting in the crosslinking densities of xanthan-chitosan hydrogel capsules decreased, amounts of bacteria would be embedded incompletely, viable counts of encapsulation and encapsulation yield of $L$. acidophilus microcapsules would be decreased, correspondingly.

As a result, there is a preliminary determination about the chitosan concentration for L. acidophilus microencapsulated. The optimum chitosan concentration was $0.9 \%$, which corresponds to viable counts and entrapped yield were $1.1 \cdot 10^{10} \mathrm{CFU} \cdot \mathrm{g}^{-1}$ and $73 \%$, respectively.

\section{Effect of xanthan concentration on encapsulation of $L$. acidophilus}

According to the initial preparation conditions of microcapsulation, xanthan concentration was adjusted to $0.5 \%, 0.7 \%, 0.9 \%$ and $1.1 \%$, the results as shown in Figure 3.

According to Figure 3, with increasing of the xanthan concentration, the viable counts and entrapped yield of L. acidophilus microcapsules increased at first, and then decreased. This phenomenon may be due to

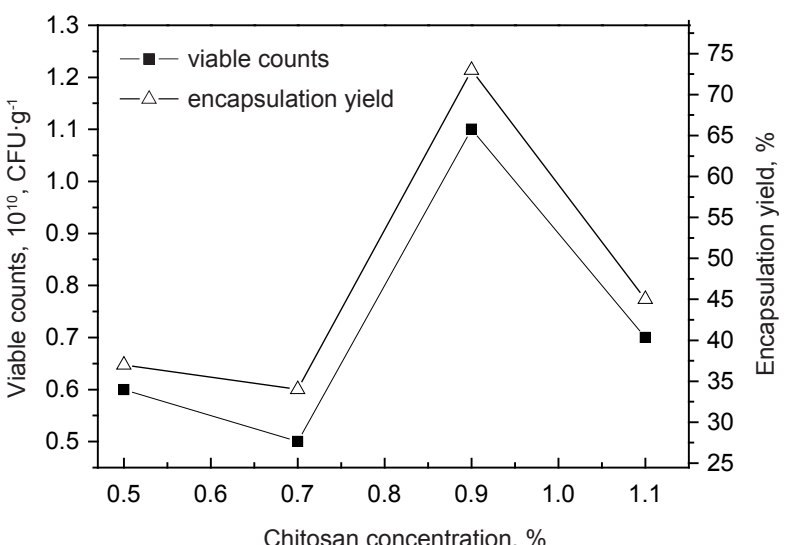

Fig. 2. Effect of chitosan concentration on viable counts and entrapped yield of L. acidophilus of microcapsules

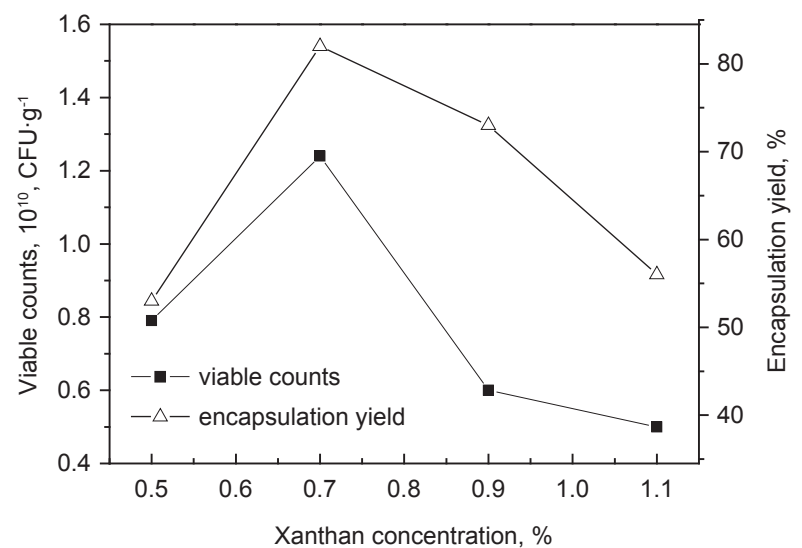

Fig. 3. Effect of xanthan concentration on viable counts and entrapped yield of L. acidophilus 1 of microcapsules 
the degree of swelling decreased when increasing the concentration of xanthan solution. When the concentration of xanthan solution increasing from $0.5 \%$ to $0.7 \%$, the crosslink density of xanthan-chitosan hydrogel capsules become increased. When the xanthan concentration was $0.7 \%$, the viable counts and entrapped yield of $L$. acidophilus microcapsules up to $1.24 \cdot 10^{10} \mathrm{CFU} \cdot \mathrm{g}^{-1}$ and $82 \%$, respectively. However, we count the number of the bacteria in a certain period of time, increasing xanthan concentration resulted in significantly greater the crosslink density of xanthanchitosan hydrogel capsules and lower the dissolution rate. As a result, it takes a lot of time to release probiotic cells under the degradation media, the viable counts and entrapped yield of L. acidophilus microcapsules will be decreased, correspondingly.

As a result, there is a preliminary determination about the xanthan concentration for $L$. acidophilus microencapsulated. The optimum xanthan concentration was $0.7 \%$, which corresponds to viable counts and entrapped yield were $1.24 \cdot 10^{10} \mathrm{CFU} \cdot \mathrm{g}^{-1}$ and $82 \%$, respectively.

\section{Effect of cell suspension-xanthan ratios on encapsulation of $L$. acidophilus}

According to the initial preparation conditions of microcapsulation, the difference proportion of prepared bacteria suspension volume $(\mathrm{mL})$ and xanthan solution volume $(\mathrm{mL})$ were investigated, such as 1:5, 1:10,

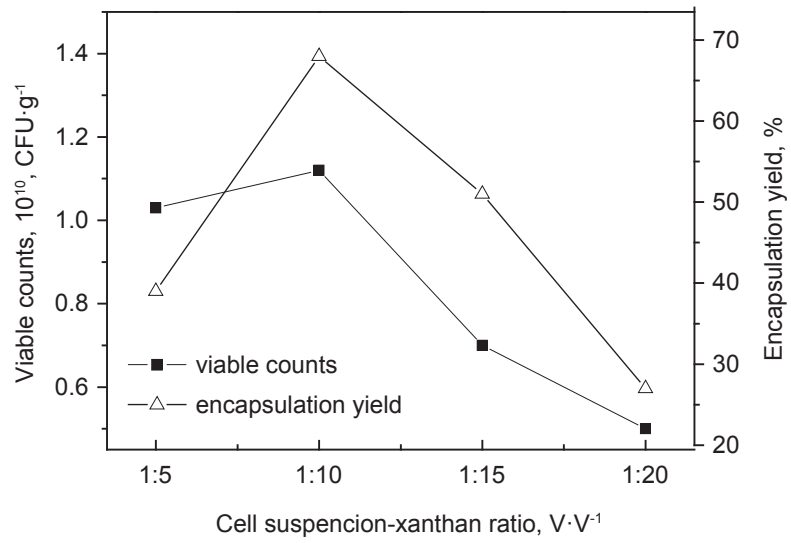

Fig. 4. Effect of cell suspension-xanthan ratios on viable counts and entrapped yield of L. acidophilus of microcapsules
1:15, and 1:20. The effect of various cell suspension-xanthan ratios on encapsulation of L. acidophilus was shown in Figure 4.

According to Figure 4, with increasing of the proportion of xanthan and bacteria suspension, the viable counts and entrapped yield of L. acidophilus of microcapsules continually increased at first, and then decreased, this phenomenon may be due to the high proportion of xanthan. With increasing the ratio of cell suspension-xanthan from 1:5 to 1:10, the crosslink density of xanthan-chitosan hydrogel capsules become increased. However, when the ratio of xanthan continually increased, and the viscosity of xanthan solution correspondingly increased, it is difficult to combine for xanthan and chitosan. As a result, the phenomenon of incomplete embedded would emerge, and most of the cells were not embedded strongly.

As a result, there is a preliminary determination about the cell suspension-xanthan ratio for L. acidophilus microencapsulated. The optimum cell suspension-xanthan ratio was 1:10, which corresponds to viable counts and entrapped yield were $1.12 \cdot 10^{10} \mathrm{CFU} \cdot \mathrm{g}^{-1}$ and $68 \%$, respectively.

\section{Effect of chitosan-mixed bacteria glue ratios on encapsulation of $L$. acidophilus}

(bacteria glue liquid-chitosan ratio is the mixtures of Xanthan gum and bacteria liquid and chitosan ratio. the purpose of this section was to study the effects of chitosan volume on the formation of microcapsules)

According to the initial preparation conditions of microcapsulation, the different proportion of mixed bacteria and xanthan volume $(\mathrm{mL})$ and chitosan volume $(\mathrm{mL})$ were adjusted to $1: 3,1: 4,1: 5,1: 6$, the results as shown in Figure 5.

According to Figure 5, with increasing of the chitosan-water ratio, the viable counts and entrapped yield of $L$. acidophilus microcapsules continually decreased. The reason of this tendency on figure was that the high value about proportion of mixed bacteria glue liquid and chitosan. With increasing of chitosan solution, chitosan solution package was thick, and the mass of microcapsule increased, so the living bacteria number contained in the unit quality of microcapsule and encapsulation yield will decrease gradually. 


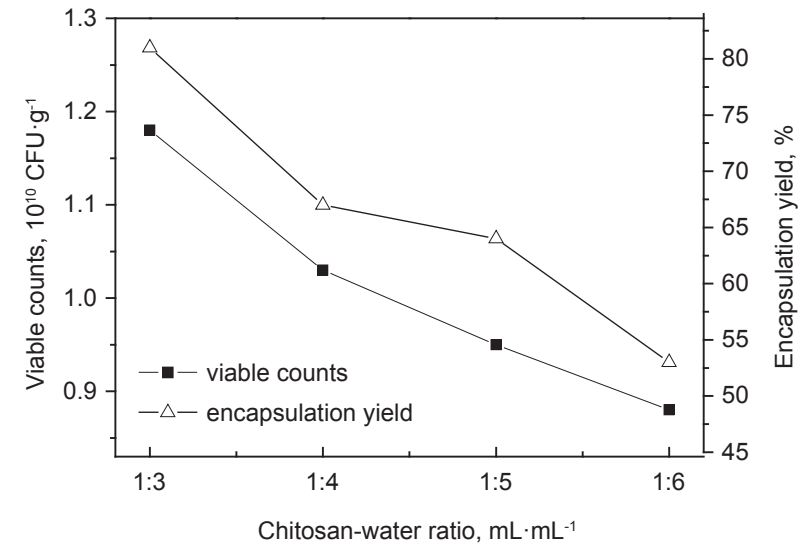

Fig. 5. Effect of e chitosan-water ratios on viable counts and entrapped yield of L. acidophilus of microcapsules

As a result, there is a preliminary determination about the cell suspension-xanthan ratio for $L$. acidophilus microencapsulated. The optimum mixed bacteria glue liquid and chitosan ratio was 1:3, which corresponds to viable counts and entrapped yield were $1.18 \cdot 10^{10} \mathrm{CFU} \cdot \mathrm{g}^{-1}$ and $81 \%$, respectively.

\section{CONCLUSIONS}

According to the above experimental study, several factors, including chitosan solution $\mathrm{pH}$ and concentration, xanthan concentration, cell suspension-xanthan ratio, mixed bacteria glue liquid-chitosan ratio, have an important influence on microcapsulation of L. acidophilus. The optimum chitosan solution $\mathrm{pH}$ for L. acidophilus was 5.5; the optimum chitosan concentration was $0.9 \%$; the optimum xanthan concentration was $0.7 \%$; the optimum cell suspension-xanthan ratio was 1:10; the optimum mixed bacteria glue liquidchitosan ratio was $1: 3$. These results will be helpful to further optimize the process of $L$. acidophilus microencapsulation, and provide reference for obtaining higher viable counts and entrapped yield of $L$. acidophilus microcapsules.

\section{REFERENCES}

Agrawal, R. (2005). Probiotics: an emerging food supplement with health benefits. Food Biotechn., 19, 3, 227-246.
Chellat, F., Tabrizian, M., Dumitriu, S., Chornet, S., Rivard, C. H., Yahia, L. (2000). Study of biodegradation behavior of chitosan-xanthan microspheres in simulated physiological media. J. Biomed. Mat. Res., 53, 592-599.

Chu, C. H., Kumagai, H., Nakamura, K. (1996). Application of polyelectrolyte complex gel composed of xanthan and chitosan to the immobilization of Corynebacterium glutamicum. J. Appl. Polym. Sci., 60, 1041-1047.

Champagne, C. P., Lacroix, C., Sodinigallot, I. (1994). Immobilized cell technologies for the dairy-industry. Critic. Rev. Biotechn., 14, 2, 109-134.

Doleyres, Y., Fliss, I., Lacroix, C. (2004). Increased stress tolerance of Bifidobacterium longum and Lactococcus lactis produced during continuous mixed-strain immobilized-cell fermentation. J. Appl. Microb., 97, 527-539.

Dutta, P. K., Dutta, J., Tripathi, V. S. (2004). Chitin and chitosan: Chemistry, properties and applications. J. Sci. Ind. Res., 63, 20-31.

Felse, P. A., Panda, T. (1999). Studies on applications of chitin and its derivatives. Bioproc. Eng., 20, 505-512.

Fuller, R. (1989). Probiotics in man and animals. J. Appl. Bact., 66, 365-378.

Gardiner, G., O'Sullivan, E., Kelly, J., Auty, M. A. E., Fitzgerald, G. F., Collins, J. K., ..., Stanton, C. (2000). Comparative survival of human-derived probiotic Lactobacillus paracase $i$ and $L$. salivarius strains during heat treatment and spray drying. Appl. Environ. Microb., 66, 2605-2612.

Garcia-Ochoa, F., Santos, V. E., Casas, J. A., Gomez, E. (2000). Xanthan gum: Production, recovery, and properties. Biotechn. Adv., 18, 549-579.

Heidebach, T., Forst, P., Kulozik, U. (2009). Microencapsulation of probiotic cells by means of rennet-gelation of milk proteins. Food Hydrocoll., 23, 7, 1670-1677.

Krasaekoopt, W., Bhandari, B., Deeth, H. (2003). Evaluation of encapsulation techniques of probiotics for yoghurt: A review. Int. Dairy J., 13, 1, 3-13.

Kimoto, H., Ohmomo, S., Nomura, M., Kobayashi, M., Okamoto, T. (2000). In vitro studies on probiotic properties of lactococci. Milchwissenschaft, 55, 245-249.

Motohiro S., Takefumi M., Masatsugu Y., Shuji A. (2009). Protection of Lactobacillus acidophilus from bile salts in a model intestinal juice by incorporation into the innerwater phase of a W/O/W emulsion. Food Hydrocoll., 23, 281-285.

Meyer, E. L., Fuller, G. G., Clark, R. C., Kulicke, W. M. (1993). Investigation of xanthan gum solution behavior under shear flow using rheooptical techniques. Macromolecules, 26, 3, 504-511. 
Chen, H., Song, Y., Liu, N., Wan, H., Shu, G., Na, L. (2015). Effect of complexation conditions on microcapsulation of Lactobacillus acidophilus in xanthan-chitosan polyelectrolyte complex gels. Acta Sci. Pol. Technol. Aliment., 14(3), 207-213. DOI: 10.17306/J. AFS.2015.3.22

Picot, A., Lacroix, C. (2004). Encapsulation of bifidobacteria in whey protein-based microcapsules and survival in simulated gastrointestinal conditions and in yoghurt. Int. Dairy J., 14, 6, 505-515.

Sandoval-Castilla, O., Lobato-Calleros, C., García-Galindo, H. S., Alvarez-Ramírez, J., Vernon-Carter, E. J. (2010). Textural properties of alginate-pectin beads and survivability of entrapped $L b$. casei in simulated gastrointestinal conditions and in yoghurt. Food Res. Int., 43, 1, 111-117.
Vinderola, C. G., Reinheimer, J. A. (2003). Lactic acid starter and probiotic bacteria: a comparative "in vitro" study of probiotic characteristics and biological barrier resistance. Food Res. Int., 36, 895-904.

Xiao Yan Li, Xi Guang Chen, Zhong Wu Sun, Hyun Jin Park, Dong-Su Cha (2011). Preparation of alginate/chitosan/carboxymethyl chitosan complex microcapsules and application in Lactobacillus casei ATCC 393. Carbohydr. Polym., 83, 1479-1485.

Accepted for print - Zaakceptowano do druku: 24.05.2015

Received - Przyjęto: 30.09.2014

For citation - Do cytowania

Chen, H., Song, Y., Liu, N., Wan, H., Shu, G., Na, L. (2015). Effect of complexation conditions on microcapsulation of Lactobacillus acidophilus in xanthan-chitosan polyelectrolyte complex gels. Acta Sci. Pol. Technol. Aliment., 14(3), 207-213. DOI: 10.17306/J. AFS.2015.3.22 